\title{
El universo femenino en la prosa temprana de Samuel Beckett: hacia la autonomía y la mundialización de la literatura
}

\author{
Eva Jersonsky \\ Universidad de Buenos Aires, Argentina
}

Copyright (c) 2015 by Eva Jersonsky. This text may be archived and redistributed both in electronic form and in hard copy, provided that the author and journal are properly cited and no fee is charged for access.

Resumen. En el presente trabajo realizaremos una lectura de More Pricks than Kicks y Dream of Fair to Middling Women, de Samuel Beckett, focalizándonos en la construcción de los personajes femeninos. Retomando algunas afirmaciones en torno a la misoginia del autor y su caracterización de las mujeres como personajes hostiles (Brienza, Kim), intentaremos apartarnos para abordar el universo femenino construido en los relatos a partir del deseo del autor de convertirse en un escritor cosmopolita y transnacional (Casanova, Even-Zohar). De esta manera, analizaremos a las mujeres que rodean la vida de Belacqua Shuah como aquellos elementos que llevan al personaje masculino a cruzar las fronteras y difuminar los límites del lenguaje, de los géneros, de las naciones y de las tradiciones literarias. Adriana Ottolenghi, Winnie, Alba, Ruby, Lucy, Thelma y Smeraldina son todas representantes de ese género femenino desplazado, como Irlanda es la desplazada de Europa, la productora de una literatura menor. No obstante, el gesto constante de Beckett es apartarse de esta tradición irlandesa, lo cual no lleva a cabo negándola o borrándola de su obra sino precisamente abriéndola a otras posibilidades, tal como estas mujeres se construyen como heterogéneas, diversas y destructoras de fronteras.

Palabras clave. Gender, Cosmopolitism, Ireland, Intertextuality, Woman.

\begin{abstract}
In the present essay we will analyse More Pricks than Kicks and Dream of Fair to Middling Women by Samuel Beckett, focusing on the construction of the female characters. Taking into account some statements regarding the author's misogyny and his characterization of women as hostile subjects (Brienza, Kim), we will try to detach ourselves from this in order to approach the female universe presented in the stories from the author's desire to become a cosmopolite and transnational writer (Casanova, Even-Zohar). In this way, we will examine the women of Belacqua Shuah's life as those elements that take the male character to cross borders and blur the limits of language, of genre, of nations, and of national traditions. Adriana Ottolenghi, Winnie, Alba, Ruby, Lucy, Thelma, and Smeraldina are all representatives of this displaced female gender, as Ireland is Europe's margin, the producer of a minor literature. Nevertheless, Beckett's constant gesture is to remove himself from this Irish tradition, which he does not carry out by denying it or erasing it from his work, but opening it to other possibilities, such as these women constituting themselves as heterogeneous, diverse, and demolishers of borders.
\end{abstract}

Key Words. Gender, Cosmopolitism, Ireland, Intertextuality, Woman.

Samuel Beckett es un autor sobre el que se han escrito una infinidad de textos tanto de análisis de su producción como de su persona. El hecho de que los críticos y especialistas se hayan 
dedicado tanto a su faceta literaria como biográfica reside principalmente en el conflicto del autor con sus raíces nacionales irlandesas y el efecto que esto ha tenido en su escritura, como señala Sontag: "one cannot use the life to interpret the work. But one can use the work to interpret the life" (1980: 111).Siguiendo esta línea, tendremos en especial consideración su conflicto con Irlanda como germen de su proyecto literario. El particular combate que entabla con su tradición literaria y con el funcionamiento del sistema literario en general lo llevan a tomar una posición crítica y experimental que no solamente puede observarse en la elección de diferentes lenguas sino también en el juego que realiza con las mismas, en los temas que elige desarrollar y en las formas literarias que adopta y adapta a sus necesidades. Sin embargo, para el autor, todos estos diferentes niveles de análisis son parte de un mismo ideal y una misma búsqueda estética que pretende romper con las fronteras nacionales, con la tradición que le correspondería y con una idea de la literatura que considera caduca. Como señala Pilling (2011), en relación a More Pricks than Kicks (MPTK), abordamos estos libros como producto de tensiones personales y genéricas (x).

En el presente trabajo nos focalizaremos en dos obras tempranas del autor, More Pricks than Kicks (1934) y Dream of Fair to Middling Women (Dream) - esta última escrita en 1932, antes de la primera pero publicada póstumamente en 1992. En estos podemos localizar el germen del proyecto estético y literario, los comienzos de una obra que desarrollará a lo largo de su vida. Este proyecto, que asociamos principalmente con las ansias de Beckett de desprenderse de sus raíces irlandesas, gira en torno a la necesidad de difuminar los límites nacionales, estéticos y lingüísticos que encierran a la literatura en un espacio agonizante. El autor, entonces, pone en funcionamiento diferentes procedimientos con el objetivo de ir más allá de las fronteras dentro de las que nació. Nuestro objetivo reside en leerlas en conjunto, sin obviar el proceso de reescritura, tomando como eje la construcción del universo femenino que configura la lengua y la forma de los textos y las acciones del personaje principal. Estas mujeres, heterogéneas y destructoras de fronteras, son las que le permitirían a Beckett dar el puntapié inicial en su carrera en contra de la tradición nacional y a favor de una mundialización o trans-nacionalización de la literatura, a la vez que vuelven a introducir el componente biográfico en el análisis, como veremos más adelante. Ellas, como personajes que vinculan uno y otro texto, son la muestra del comienzo de una obra que pretende salir de Irlanda a Europa y de allí al mundo.

\section{Irlanda: recuperación y ruptura}

Con respecto a lo anteriormente dicho, Sean Kennedy claramente señala este fenómeno al afirmar que:

\section{future accounts of Beckett's aesthetic develop- ment must come to terms with the enduring impact of his early filiations with Ireland. The same accounts will have to allow, of course, for the ways in which those filiations were resented and resisted, and, at various times, largely superseded by other concerns (2010: 1).}

Y no es el único autor que lo ha señalado, Pascale Casanova sostiene que Beckett se constituye en contra de su patrimonio literario y lingüístico nacional (2001: 63), combatiendo el monopolio del inglés en Irlanda (81) y viajando a París para afirmar "la autonomía total de la literatura" (173). Como mencionamos con anterioridad, "[p]or un conducto específicamente literario" Beckett rechaza "las normas nacionales que estaban vigentes en Irlanda" (423), su proyecto estético es el que le permite separarse de su tradición. Sin embargo, y como sostiene muy acertadamente Kennedy: "Beckett was not a modernist "despite" his Irish roots but precisely because of them" (2010: 7), es decir que el desarrollo de su obra no puede ser pensado como un corte total con respeto a sus raíces sino precisamente como una construcción que requiere de estas para tomar forma. "The two spaces of his writing, Ireland and Europe, need to be considered together" (7), afirma el autor más adelante, y aquí encontramos la base de nuestra hipótesis, la obra de Beckett no puede ser leída dejando de lado su herencia irlandesa, incluso en la forma de un rechazo y degradación, ni dejando de lado su afán por conquistar una Europa total. La autonomía que el escritor tanto ansía nace de una tradición literaria que lo ahoga y busca en otros territorios y lenguajes la libertad de producir por fuera de sus fronteras. 
Más allá de la opinión de los diferentes críticos que se han interesado en la obra y vida de Samuel Beckett es el autor mismo quien ha hecho explicitas, a su manera, diferentes posiciones con respecto a su idioma y su nación. En la German Letter of 1937 señala el problema de la lengua materna al decir: "[a]re you bored by reading my German letters as I am in writing one in English?" (171), y al expresar la dificultad de mantenerse dentro de los límites del idioma: "[i]t is indeed becoming more and more difficult, even senseless, for me to write an official English. And more and more my own language appears to me like a veil that must be torn apart in order to get at the things (or the Nothingness) behind it" (171). Algo se oculta detrás de esta lengua materna oficial, por eso será su misión estética y literaria descubrir otros lenguajes y otros territorios para que su obra obtenga una mayor autonomía.

Retomando algunas ideas expresadas al comienzo de este trabajo, el análisis se realizará no solamente considerándolos como representantes individuales de los comienzos del proyecto beckettiano sino también en conjunto como pruebas del proceso de recuperación y reescritura que el autor ha llevado adelante a lo largo de toda su carrera. Este procedimiento es característico del autor, tanto con respecto a sus propios textos como a producciones ajenas, y forma parte de su búsqueda de autonomía y ruptura de fronteras lingüísticas, estéticas y literarias. En este sentido, encontramos a Dream of Fair to Middling Women, una novela que no se publica en vida del autor debido principalmente a la falta de editores dispuestos a hacerlo, de la cual algunas historias son recuperadas en la colección de cuentos More Pricks than Kicks un par de años después. Aquí se encontraría la primera ambigüedad y juego del autor ya que, como señala Gontarski con respecto al conjunto: "with its single, unifying character, Belacqua Shuah, is as much a novel as a collection of stories" (1995: xiii). Entonces, el movimiento se realiza de una novela, en la que ya hay signos de ruptura con el género hacia una colección de historias que puede ser tanto esto como una novela, o un libro experimental (Pilling 2011: 16). El velo del idioma inglés que Beckett sostenía que debía romper también puede pensarse en relación a los géneros, como señala Tajiri (1994), por ejemplo, en relación a Dream como mezcla de lenguajes y estilos (71).

Estas historias y la recuperación con respecto a la novela anterior toman como eje el universo femenino que rodea al personaje principal, Belacqua Shuah. Diferentes mujeres, representado tal vez la diversidad del mundo que ansiaba el autor, desfilan por su vida y configuran su accionar. Algunas de ellas, como Smeraldina o Alba, se repiten en fragmentos que llegan incluso a ser idénticos. Estas mujeres, estas historias, son definidas como: "the result of a process by which a young writer trapped in a suffocating environment exorcised his hatred and fears" (Fernández 2012: 94), son la forma que el autor encontró para comenzar su búsqueda de ese otro lugar por fuera del sistema literario nacional. Sin embargo, Gontarski señala: "althought selfconsciously experimental, self-referential, and often mannered, Beckett's short fiction is never wholly divorced from the culturally pervasive traditions of Irish storytelling" (1995: xii), el autor coloca su mirada en París, en una forma diferente de expresarse pero sus raíces irlandesas siempre encuentran la manera de colarse dentro de su escritura, por eso mismo, según este autor: "Beckett's short prose can span the gulf between the more fabulist strains of Irish storytelling and the aestheticized experimental narratives of European modernism" (Gontarski 1995: xii). Este oscilar entre Irlanda y Europa, entre las raíces y la autonomía literaria, también se multiplica en los diferentes niveles de su producción, como señala Gontarski: "much of Beckett's short prose inhabits the margins between prose and poetry, between narrative and drama, and finally between completion and incompletion" (1995: xxi).

Como señala Katz en un texto en el que lleva a cabo un análisis de la primera historia de MPTK: "une langue étrangère crée une rupture dans la continuité subjective. Cette rupture est une violence que Beckett reconnaissait et même appréciait, sans doute parce qu'il ressentait ce déchirement même à 'l'intérieur' de sa première langue" (2000: 224). La utilización de otras lenguas y la distorsión de las mismas, fenómenos que se repiten constantemente en Dream y en MPTK, son uno de los aspectos, que junto con la ruptura de los géneros y de los estereotipos temáticos, le permiten a Beckett 
crear una grieta que le brinda una salida de los límites heredados. En Dream, por ejemplo, Smeraldina tiene varios problemas con el idioma inglés, desde no poder entender palabras, como "monologue" (1993: 25) o "surreptitious" (95) hasta el hecho de que su lamento sea descripto como alemán (1993: 30), incluso sus cartas son un híbrido entre el inglés y el alemán (1993: 55-61).

Luego, las descripciones y comentarios que se hacen sobre Ginette son en francés (1993: 143-144) y Alba emite frases, en general sumamente coloquiales, en español como "hijo de la puta blanca" y "carajo" (1993: 151, 153). Por otro lado, se puede mencionar la presencia de palabras distorsionadas o con una escritura diferente de la de la norma estándar como "wunnerful" (151). Como podemos observar, en general aquellas transformaciones y mezclas de lenguajes giran en torno a las mujeres. Esto mismo se repite en MPTK, tanto Smeraldina como Alba vuelven a aparecer como elementos disruptivos. A estas se suma la presencia del italiano a través de la professoressa Ottolenghi en "Dante and the lobster", el hecho de que Alba sueñe en francés (1972: 81-82) y el dialecto particular de Ruby (1972: 95), entre otras.

En un ensayo anterior ya Beckett señalaba su conflicto con la lengua, especialmente con el inglés. En "Dante...Bruno. Vico...Joyce" sostenía: "no language is so sophisticated as English, It is abstracted to death" (28) y posicionaba al inglés con respecto al resto de las lenguas europeas en comparación al latín medieval con respecto a los dialectos italianos (30). Su lengua materna era un ancla para su escritura y estos textos muestran un gran avance con respecto a la ruptura buscada. Otro punto interesante es la afirmación de que forma y contenido son una misma cosa (27), lo cual nos remite nuevamente al principio de este trabajo donde señalábamos los diferentes niveles de análisis, aclarando que para el autor estos funcionan como uno solo. Dentro de su proyecto la forma adoptada, la ruptura genérica, la distorsión lingüística y la configuración de los personajes son parte de un mismo proceso.

\section{Recuperación y reescritura}

Y si nos referimos al proceso de escritura debemos retomar la reutilización de diferentes fragmentos que lleva a cabo Beckett en sus textos. El caso que tomamos es bastante claro debido a la presencia de un grupo de personajes recurrentes y escenas que se repiten palabra por palabra o de manera similar. De hecho, muchas de las partes de MPTK provienen de diferentes escritos o publicaciones anteriores, entre ellas Dream. ${ }^{1}$

Para comprender un poco más de qué estamos hablando cuando nos referimos a la reutilización y reescritura, podríamos recurrir a Gerard Genette. Él explica un procedimiento bien amplio que denomina transtextualidad en la que un texto (hipotexto) es transformado en otro (hipertexto) (1989: 17). Y, a su vez, sostiene que "todas las obras son hipertextuales" (19). Todo texto remite a otro y, en el caso de Beckett, a veces de manera muy explícita. Además, en todo hipertexto hay una cierta ambigüedad que "proviene precisamente de que un hipertexto puede a la vez leerse por sí mismo y en su relación con el hipotexto" (1985: 113). Esta "duplicidad de objeto" la describe como un palimpsesto, "donde sobre un mismo pergamino se ve un texto que se superpone a otro, no del todo disimulado sino que se ve por transparencia" (114). Dream sería uno de los hipotextos del hipertexto MPTK y, aunque ambos pueden leerse por separado, siempre vamos a encontrar la imagen del texto anterior cuando leamos la recopilación de relatos. A esto nos referimos específicamente cuando hablamos de leerlo en conjunto, es decir, como dos pasos de un mismo proceso. La reescritura y reutilización de un mismo material le permite a Beckett relativizar el texto mismo y acercarse un poco más al ideal de autonomía y mundialización.

El hecho de que haya una cierta continuidad entre los relatos de MPTK es parte de la herencia de Dream, como señala Fletcher:

[i]t should be remarked that although the ten stories, arranged in chronological order, are not explicitly linked (transitions from one to the next are often abrupt), the book as a whole is carefully constructed, and the various parts, often different in style, theme and tone, harmonize to give a fairly coherent impression of a certain man's life (1965: 20-21).

1. Este proceso es explicado más detalladamente por otros autores, como Cassandra Nelson (2010: ix). 
Incluso podemos encontrar ciertos comentarios o notas al pie insertados en el texto que remiten a diferentes partes, vinculándolas entre sí. Por ejemplo, en una nota al pie de "Walking out" leemos: "Cp. Fingal" (1972: 112), nótese especialmente la denominación Cp. o chapter, "capítulo" en vez de "relato" o algún sinónimo de este. Este fenómeno se repite en "What a misfortune" donde observamos la siguiente nota: "Cp. Walking out" (1972: 150). Por último, en este mismo relato o "capítulo" encontramos una referencia: "the nice Little girl in A wet night" (1972: 126-127).

De la novela al conjunto de relatos encontramos una primera transformación. Luego, la problemática respecto al mundo femenino se expande. En MPTK este universo se agranda y son más y más diversas las representantes del género femenino que marcan su camino. Esta recurrencia también es explicitada por Fletcher:

[1]ater, Belacqua asserts there is no such thing as love in a thalamus, and in More Pricks than Kicks the same idea of the incompatibility of love and sex is insisted upon. Lucy must take a cicisbeo, Ruby has a difficult task in inducing Belacqua to take her in his arms, and Thelma finds his indifference bewildering. Throughout the Dream, too, his so-called bloodless nonchalance is emphasized. His most real self, we are told, is that of Belacqua the Florentine, the next most real Narcissus fleeing Echo, the least real Apollo seeking Daphne (1965: 53).

Belacqua se encuentra en un movimiento constante de atracción y repulsión, siempre en torno a estas mujeres que teme y ama. Finalmente el problema del protagonista es similar al que atormenta al escritor:

[ $\mathrm{t}$ ]he spiritual problem (the last significant motif) arises from Belacqua's need to shut himself off from the outside world, to achieve selfsufficiency (that self-sufficiency which, we are told, he never wearied of arrogating to himself), to dam himself up against the inroads the surrounding world makes into him (Fletcher 1965: 55).

Mientras que Belacqua quiere una independencia que jamás consigue, Beckett quiere una autonomía para la literatura que parece imposible, Belacqua se puede pensar como alter ego de este autor (Pilling, 2011: 16). No obstante, lo que el protagonista pretende alcanzar en su soledad, Beckett lo busca en la apertura al mundo, la literatura en su independencia de las raíces nacionales pero en conexión con una diversidad europea o mundial, como la del universo femenino que rodea a Belacqua.

Este proceso de transformación y recuperación de su propia escritura es un gesto que va en la misma dirección que la auto-traducción. De esta forma, los textos de Beckett intentan depender solo de él mismo y no de ninguna literatura nacional y por ser solo de él mismo pueden ser de cualquier lugar. Este es el objetivo también de Belacqua.

\section{Sistema literario mundial}

Antes de continuar consideramos necesario ahondar un poco más en esto que denominamos mundialización de la literatura. Para esto recurriremos a la teoría de los polisistemas de Even-Zohar y a la idea de La República mundial de las Letras de la autora ya mencionada Pascale Casanova.

En primer lugar, Itamar Even-Zohar en Polysystem studies sostiene que la literatura no es una actividad aislada de la sociedad sino un integrante $y$ un factor poderoso en las actividades humanas (1990: 2). Esta premisa es importante para nuestro trabajo ya que desde el comienzo estamos afirmando la importancia que tiene el factor irlandés. La literatura es, entonces, parte de la sociedad en la que se encuentra inmerso el autor. A partir de esta idea es que Even-Zohar desarrolla un concepto innovador como el de polisistema: "a polysystem - a multiple system, a system of various systems which intersect with each other and partly overlap, using concurrently different options, yet functioning as one structured whole, whose members are interdependent" (1990: 11). A su vez, es importante recordar que los sistemas no son iguales sino que tienen jerarquías y por esto mismo conforman un sistema dinámico y en lucha permanente (14). Estas características nos remiten, por un lado, a estas mujeres que podrían ser leídas como los diferentes sistemas que giran en torno a Belacqua y hacen de su vida un polisistema heterogéneo - en palabras del mismo Belacqua: "heterogeneous entities" (1993: 35). Por otro lado, también se pueden leer en relación al conflicto de Beckett con sus raíces irlandesas y su llegada a la literatura europea. Él no se conforma con ocupar un lugar estanco en uno de los sistemas nacionales sino que su objetivo 
es poder navegar este sistema europeo o mundial, antes que irlandés.

Por otro lado, Casanova comparte algunos puntos con Even-Zohar pero se concentra de forma más marcada en el sistema literario tomando como ejemplos a algunos escritores que han cruzado fronteras, como es el caso de Beckett. Para la autora la relación entre los textos y el sistema literario mundial se define a partir de lo siguiente:

cada libro escrito en el mundo y declarado literario sería una ínfima parte de la "combinación" de toda la literatura mundial. Lo que podría, pues, parecer lo más ajeno a la obra, a su construcción, a su forma y su singularidad estética, es, en realidad, lo que engendra el propio texto, lo que permite que surja. Es la configuración o la composición del conjunto de la alfombra, es decir, en el orden literario, la totalidad del "espacio literario mundial", lo único que puede conferir sentido y coherencia a la forma misma de los textos (2001: 14).

A esto se le suma la idea recurrente de la lucha, en este caso, entre lenguas rivales (14). Las identidades y literaturas nacionales, entonces, no son ajenas a este sistema pero se encuentran en lucha permanente generando un sistema dinámico y jerárquico, como el presentado por Even-Zohar, donde algunos escritores y algunas obras pueden salir de su compartimento y participar del movimiento y la diversidad propios de una literatura transnacional. De hecho, Beckett es uno de estos escritores que "pueden entonces negarse, tanto colectiva como individualmente, a aceptar la definición nacional y política de la literatura" (59). Esta negación comienza en sus textos y eso es lo que observamos en la lectura de Dream y MPTK.

Casanova también señala que "cada escritor no hereda de la misma forma su pasado literario" y que Beckett se constituye en contra de él (63). Su relación con la patria es "desgraciada, excesiva y, sin embargo identificativa" (Casanova, 2001: 240). Huyendo de "la implantación de un arte nacional" es que el autor se exilia en París "para afirmar, contra un arte sometido a los designios nacionales, la autonomía total de la literatura" (Casanova, 2001: 173). Este es su objetivo y, como explicita Casanova, "la autonomía, siempre relativa, se convierte en uno de los principios que ordenan el espacio literario mundial" (120).
De aquí nace el objetivo del presente trabajo: intentar una lectura de textos tempranos de Beckett en los que se plasman las ansias de liberación del espacio nacional para pasar a un espacio mundial, cómo "por un conducto específicamente literario" se puede ver "el rechazo de las normas nacionales que estaban vigentes en Irlanda" (Casanova, 2001: 423), cómo

su búsqueda cada vez más rigurosa y precisa de una autonomía radical le induce a romper con todas las formas de dependencia nacional propias de los escritores: la nación en el sentido político, por supuesto, pero más aún los debates propios de la historia literaria nacional, las opciones estéticas dictadas por el espacio literario nacional $\mathrm{y}$, por último, la lengua misma concebida como un conjunto de leyes y de normas impuestas (Casanova, 2001: 445).

\section{El universo femenino}

Las mujeres en su conjunto, tanto de Dream como de MPTK, conforman uno de estos niveles que le permiten a Beckett romper con las imposiciones nacionales y acercarse a una mayor autonomía literaria. A su vez, derivan de muchas de sus experiencias personales. Algunas de las que señala Knowlson (2004) y que consideramos en relación a las obras analizadas son: Smeraldina en paralelo a su prima, Peggy Sinclair, de quien se enamora y con quien inicia su experiencia sexual; Syracusa como una reconstrucción de Lucia Joyce, hija de su mentor, con quien entabla una relación tormentosa; Alba, como aquella a quien ama pero con quien no puede lograr la unión, representación de Ethna MacCarthy; entre otras. El autor se siente atraído por una cantidad considerable de mujeres que desfilan por su vida, pero esto no lo vive con satisfacción sino como un sufrimiento y una debilidad de la que desearía poder librarse. La intelectualidad es su consuelo, en constante conflicto con la sensualidad femenina que lo atrae, y esta lucha atraviesa abiertamente estos textos de juventud.

Como señala Kim, la ficción temprana de Beckett: "reveals intricate correlations between the representations of Ireland and women" (2005: 57). La primera identificación que podemos llegar a realizar, entonces, es entre el universo femenino e Irlanda. Sin embargo, es una relación más compleja de la que parece a simple vista, ambos son elementos desplazados 
dentro de diferentes sistemas. Por un lado, el género femenino es históricamente marginado, considerado de segundo grado, débil y sumiso. Por otro lado, Irlanda es una nación desplazada dentro del sistema europeo, se constituye como margen y está sometido a otras naciones más poderosas, ya desde el plano lingüístico. $\mathrm{Su}$ literatura es lo que Deleuze y Guattari han denominado "literatura menor": "literatura que una minoría hace dentro de una lengua mayor" (1978: 28). Siguiendo este razonamiento, el gesto de Beckett podría leerse como el intento de llevar a Irlanda al mundo, de diversificarla y liberarla de sus ataduras, no como un intento de emancipar la literatura irlandesa sino como una ambición literaria individual que toma como punto de partida una tradición que se le ha impuesto. El escritor irlandés es capaz de independizarse e independizar su literatura de los mandatos nacionales. Las mujeres, heterogéneas, diversas, representantes de diferentes espacios y lenguas, son la nación ideal que, paradójicamente, rompe con las fronteras nacionales. ${ }^{2}$

El universo femenino es el que lleva al hombre a cruzar fronteras, tanto literal como figuradamente, y al ser este grupo tan heterogéneo el territorio recorrido por el protagonista es completamente diverso y ecléctico. Brienza, por ejemplo, sostiene: "Beckett's characterization of women alternates between stereotypes of femininity and bizarre reversals of the stereotypes" (1992: 91), es decir, ambas caras de la moneda están representadas en su obra. No obstante, el argumento de esta autora se construye en torno a un Beckett misógino y no es precisamente este camino el que seguimos aquí. Aunque no podríamos atrevernos a afirmar que la visión beckettiana de las mujeres es bondadosa tampoco nos resulta productivo pensar en la idea del personaje femenino solamente como depositario de un odio o desprecio genérico. Mientras Brienza sostiene que en la obra del autor irlandés lo que predomina es: "the idea that woman as a clod of earth impedes intellectual man" (91), aquí preferimos rescatarlas

2. Con respecto a esto último no podemos dejar de lado la larga tradición de identificación de la figura femenina con la nación, lo cual señala también Kim en relación a la obra de Beckett (2005: 59). como aquellas que en su banalidad y sensualidad, en su cualidad de seres puramente terrenales y físicos, le permiten al protagonista liberarse de su intelectualidad que aún se encuentra atada a un sistema de reglas que le impide cruzar ciertas fronteras. Estas mujeres, como en un nivel más general son los textos, poseen un poco de esa Irlanda y un poco del mundo. Existe una parte hostil que corresponde a esa patria conservadora que se ataca (Kim, 2005: 60) pero también otra que le permite al autor acercarse a su meta.

La heterogeneidad a la que retornamos una y otra vez se nos presenta en el sentido de que cada mujer es descrita de manera particular, cada una diferente a la anterior, representantes de diferentes lenguajes, estilos, virtudes o defectos. Al mismo tiempo, los textos se encargan de aclararnos que estas son algunas representantes de este universo, pero como son estas podrían ser otras, así es como leemos en Dream: "the Smeraldina-Rima or any other girl for that matter" (1993: 9). Y, a su vez, que la intención es que los personajes sean representantes de otra cosa, agentes en el gesto beckettiano: "it is to be hoped that we can make them stand for something (1993: 9). Las mujeres son las que mueven los hilos: "a man knows but a woman knows best" se nos aclara (1993: 19).Y el centro es Belacqua ("love demands narcissism" (1993: 39)) quien danza al compás femenino: "he had no choice but to follow her out" (1993: 91).

$\mathrm{Si}$ nos concentramos específicamente en los personajes femeninos, en primer lugar deberíamos mencionar a Smeraldina-Rima, asociada al mundo de la música (1993: 3, 13). La primera impresión que posee Belacqua de ella en Dream es poco confiable ya que él se encuentra cansado y por eso ve lo siguiente: "her face more beautiful than stupid" (1993: 3), al mismo tiempo esa estupidez es reforzada por este hecho: "she cared for nothing in heaven above or the earth beneath or the waters under the earth so much as the music of Bach" (1993: 3). Ella es la primera en esta larga línea de seres terrenales que parecen contrastar con la idea de intelectualidad y autosuficiencia que busca el protagonista. Belacqua, frente al viaje de Smeraldina, comienza a cruzar una de las fronteras cuando oscila entre la "Smeraldinalgia" y lo que se define como: "the much greater affliction of being a son of Adam" 
(1993: 5). La partida de la mujer a Alemania, entonces, no solo lo lleva a desplazarse físicamente y tener que lidiar con otros lenguajes [el musical y el alemán (1993: 30)] sino también a tener un pensamiento dinámico: "the Smeraldina-Rima is not demonstrable. She has to be taken or left. Belacqua did a Little of both" (1993: 13).Incluso la descripción misma de su cuerpo lo lleva a instalarse en otro espacio estético al hacer referencia a: "Botticelli thighs" y la "Madonna Lucrezia del Fede" (1993: 15). Smeraldina en su totalidad se encarga de que el protagonista nunca pueda quedarse cómodamente en un estado, profanando constantemente su mente y su cuerpo, incluso llegando a una violación (1993: 18). Además, este personaje es realmente uno de los más productivos, especialmente de Dream, debido no solo a sus excentricidades y movimientos geográficos sino también al desafío constante que representa en su interacción con Belacqua. Ella pone continuamente en crisis el discurso del protagonista, ya sea debido a que niega conversaciones anteriores ["I never agreed with anything, you never said such horrid things to me" (1993: 25)] o porque no puede mantenerse dentro de los límites de un lenguaje (1993: 26, 30, 5561). Más tarde, el encuentro con otra muchacha, Miranda, solo refuerza esta idea de movimiento ya que el intercambio lo lleva a la siguiente situación: "he did not want to be slavered and slabbered on by her, he thought it would be nice to be slavered and slabbered on elsewhere for a change. So he packed a bag and made to depart" (1993: 12).

Otro personaje presente en ambas obras es Syra-Cusa. Esta, en Dream, contrariamente a Smeraldina posee un cuerpo perfecto (1993: 33) pero, al mismo tiempo, leemos: "her head was null" (1993: 33). En este sentido, una nueva mujer es un nuevo desplazamiento, lingüísticamente del alemán de Smeraldina pasa al francés: "perhaps only the french can do it. Perhaps only the french language can give you the thing you want" (1993: 48) y, más adelante: "I don't know how to write a stinger in English, I always overdo it. In French I can write a fine stinger, but in English I overdo it" (1993: 64). Es precisamente en este momento donde se comienza a plantear el trazado de un sistema, de Smeraldina-Rima a Syra-Cusa la red empieza a armarse y el sistema adquiere movimiento: "the Smeraldina-Rima and the Syra-Cusa were related and compared, just as Lucien may later enter the scales with Chas" (1993: 34). Una cadena comienza a formarse: "we could chain her up [the Syra-Cusa] with the Smeraldina-Rima and the little Alba, our capital divas, and make it look like a sonata" (1993: 49). Y la ley de la proliferación de la que hablaba Even-Zohar se activa: "because his soul, by implication, had as many brides as his body" (1993: 40).

Esta tercera diva, Alba, "derelict daughter of kings" (1993: 54) representa otro viaje:

\begin{abstract}
from the merely snout-fair Smeraldina, that petulant, exuberant, clitoridian puella, who has not the first glimmering of an idea of how to set her cold bath on fire, whom now it is high time to turn round and dislike intensely, like collops of pork gone greasy, to Alba, Alba, royal diminutive, $\mathrm{Du}$, dust of a dove's heart, the eyes the eyes black till the plagal east shall resolve the long night-phrase (1993: 111).
\end{abstract}

En Alba parece estar el ideal, la necesaria liberación del individuo: "he was blind to the charms of the mighty steaks and jug-dugs of the Smeraldina-Rima and angered by the Priapean whirlijiggery-pokery of the Syra-Cusa because in both cases he was disarmed, he was really unable to rise to such superlative carnal occasions" (1993: 137). Con respecto a lo anteriormente expuesto una idea de Ackerley se nos presenta como particularmente pertinente. Él sostiene: "Belacqua's misogyny in Dream is as much self-laceration as it is flagellation of the fairer sex" (2002: 55). En nuestra línea de análisis, este ataque a sí mismo se encuentra relacionado con el rechazo de su propia parte irlandesa que continúa apareciendo. Esta frustración frente a Smeraldina y Syra-Cusa parece ir en consonancia con ese desprecio por su origen que, irónicamente, se encuentra representado en su signo contrario (conservadurismo irlandés vs. Carnalidad). No obstante, Alba es otro eslabón de la cadena. Alba también es un estereotipo, una "woman of Spain" (1993: 195), un viaje a otros lenguajes (1993: 151, 153).

Por último, Frica se nos presenta como una crisis del territorio y del lenguaje desde otro lugar: "tricks and turns and games were food and drink to the Frica" (1993: 224) y más adelante: "there was no nonsense about the Frica. When she meant skin, she said skin" 
(1993: 230). En este caso una mujer que oscila entre la más rigurosa significación de las palabras y los juegos y trucos como una forma de vivir.

Finalmente, en Dream, el centro que creíamos representado por Belacqua alrededor del cual el sistema femenino tomaba forma parece ser múltiple llevando hasta sus límites la proliferación: "there is no authority for supposing that this third Belacqua is the real Belacqua any more than that the Syra-Cusa of the abstract drawing was the real Syra-Cusa" (1993: 121).

Por otra parte, en algunas de las lecturas mencionadas referidas a las tres "divas" principales son válidas, especialmente en lo referente a los episodios "A wet night" y "The Smeraldina billet doux". Sin embargo, como señalamos con anterioridad, el conjunto de mujeres representadas es mucho más amplio, como si se intentara reforzar la idea primaria de la novela. En primer lugar encontramos a la professoressa Ottolenghi, en "Dante and the lobster" quien introduce toda la fascinación por Dante y el idioma italiano. A su vez, en esta historia podemos observar una serie de juegos en relación al lenguaje, el más pertinente para nuestra lectura sería tal vez el hecho de que se haga referencia a los distintos espacios donde se brindan las lecciones tanto de italiano como de francés y alemán. En este sentido, en este relato claramente la prioridad la tiene el italiano, frente a un alemán del cual se dice: "God knows where the German room was. Who cared about the German room anyway?" (1972: 17-18).

Por otro lado encontramos otras figuras, como Winnie que se describe como: "was pretty, hot and witty, in that order" (1972: 23). A pesar de esta descripción, esta mujer también desencadena el desplazamiento de Belacqua ya que sigue siendo una persona atada a Irlanda: "The Dublin mountains - she said - don't they look lovely, so dreamy" (1972: 23). Él, en esta situación mira en la dirección contraria y más adelante le propone: "be Roman, and we'll go on across the estuary" (1972: 22). En "Love and lethe" nos topamos con Ruby que, al igual que Smeraldina, es comparada con representantes del arte italiano (1972: 87) y se desvía de la norma del lenguaje (1972: 95). La historia de Ruby incluso termina con una frase en un francés defectuoso: "l'Amour et la Mort - caesura n'est qu'une mesme chose" (1972: 99). La siguiente mujer, Lucy, también posee elementos de Smeraldina, especialmente si nos referimos al final del episodio donde vuelven a intersectarse la música y el alemán (1972: 113).

En el episodio final, "Draff", se produce una recapitulación de las diferentes mujeres que pasaron por la vida de Belacqua:

This particular Mrs Shuah, as stated thus far at all events, does not sound very like Thelma née bboggs, nor is she. Thelma née bboggs perished of sunset and honeymoon that time in Connemara. Then shortly after that they suddenly seemed to be all dead, Lucy of course long since, Ruby duly, Winnie to decency, Alba Perdue in the natural course of being seen home. Belacqua looked round and the Smeraldina was the only sail in sight (1972: 175).

$\mathrm{Y}$ es como si este final enlazara con el principio de Dream, la vuelta a Smeraldina, el sistema que se alimenta a sí mismo y continúa en un movimiento perpetuo. Belacqua finalmente fallece, pero el sistema heterogéneo que dinamizaba su vida parece que nunca se detiene, ya sea en forma de estas mujeres particulares o de otras. Y es precisamente ese el sistema literario al que aspira Beckett, abierto, heterogéneo, diverso, en constante movimiento, tan autónomo que puede existir sin la presencia de su centro.

\section{Conclusión}

En su afán por liberarse de las cadenas irlandesas y alcanzar la autonomía literaria, Beckett comienza un proyecto estético que cambiará las reglas del espacio literario En estos textos, atravesados por un importante proceso de reescritura y reutilización, hay un elemento que se repite y cobra especial importancia: a pesar de la advertencia: "the only unity in this story is, please God, an involuntary unity" (1993: 133), el universo femenino y su interacción con el protagonista es el elemento central de ambas historias. Estas mujeres funcionan como un sistema en continuo movimiento $\mathrm{y}$, de este modo, se comportan como un modelo para la gran idea de Beckett con respecto a la literatura. Esta idea, la de un espacio literario mundial y una literatura transnacional y autónoma, es tan conflictiva como la relación de Belacqua con 
las mujeres, es un cruce de fronteras que permanentemente oscila entre la patria, esa Irlanda que al final de Dream encuentra su encanto en la lluvia (1993: 240) y el mundo, todas esas culturas que Beckett anhela y que su universo femenino ficcional representa.

\section{Bibliografía}

Ackerley, Chris. 2002. "LASSATA SED: Samuel Beckett's Portraits of his Fair to Middling Women". Samuel Beckett Today/Aujourd'hui. Volumen 12, 55-70.

Beckett, Samuel. 1972 [1934]. More Pricks than Kicks. New York: Grove Press. .1983. "Dante...Bruno. Vico...Joyce". Disjecta. Miscellaneous Writings and a Dramatic Fragment. London: Calder, 19-33. .1983. "German letter of 1937". Disjecta. Miscellaneous Writings and a Dramatic Fragment. London: Calder, 171-173. 1993. Dream of Fair to Middling Women. London: Calder.

Brienza, Susan. 1992. "Clods, whores, and bitches. Misogyny in Beckett's early fiction". Ben-Zvi, L. (ed.), Women in Beckett: Performance and Critical Perspectives. Urbana and Chicago: University of Illinois Press, 91-105.

Casanova, Pascale. 2001. La república mundial de las Letras. Barcelona: Anagrama.

Deleuze, G. y Guattari, F. 1978. “¿Qué es una literatura menor?”. Kafka. Por una literatura menor. México: Era, 28-44.

Even-Zohar, Itamar. 1990. "Polysystem studies". Poetics Today. Volume 11: $\mathrm{n}^{\circ} 1$.

Fernández, José Francisco. 2012. “'So it goes in the world”: the politics of Samuel Beckett's More Pricks than Kicks". Martín Alegre, S. (coord. y ed.), Moyer, M., Pladevall, E. y Tubau, S. (eds.), At a time of crisis: English and American studies in Spain. Barcelona: Universitat Autònoma de Barcelona/AEDEAN, 94-99.

Fletcher, John. 1965. The novels of Samuel Beckett. New York: Barnes \& Noble.

Genette, Gerard. 1985. "La literatura al segundo grado". Maldoror. Volumen 20, 101-116. .1989. "Capítulo I". Palimpsestos. Madrid: Taurus, 9-17.

Gontarski, S. E. 1995. "Introduction. From Unabandoned Works: Samuel Beckett's Short Prose”. Beckett, S., The complete short prose of Samuel Beckett, 1929-1989. New York: Grove, xi-xxxii.

Katz, Daniel. 2000. "Beckett et les huit langues". Samuel Beckett Today/Aujourd'hui. Volumen 10, 223-229.

Kennedy, Sean. 2010. "Introduction. Ireland/Europe... Beckett/Beckett". Beckett and Ireland. Cambridge: Cambridge University Press, 1-15

Kim, Rina. 2005. "Severing connections with Ireland: Women and the Irish Free State in Beckett's writing". Beckett Today/Aujourd'hui. Volumen 15, 57-69.

Knowlson, James. 2004. Damned to Fame: The Life of Samuel Beckett. New York: Grove.

Nelson, Cassandra. 2010. "Preface". Beckett, S., More Pricks than Kicks. London: Faber \& Faber, vii-xxi.

Pilling, John. 2011. Samuel Beckett's 'More Pricks than Kicks': In a Strait Of Two Wills. London: Continuum.

Sontag, Susan. 1980. Under the sign of Saturn. Toronto: McGraw-Hill Ryerson.

Tajiri, Yoshiki. 1994. "An introduction to Beckett's Dream of Fair to Middling Women". Hitotsubashi Journal of Arts and Sciences. $\mathrm{N}^{\circ} 35,71-83$.

Received $30^{\text {th }}$ October 2014 Last version $10^{\text {th }}$ January 2015

Eva Jersonsky es licenciada y profesora en Letras, egresada de la Universidad de Buenos Aires, especialista en literatura española contemporánea. En la actualidad disfruta de una Beca Estímulo de la UBA y participa en un grupo de investigación UBACyT focalizado en la trans-nacionalidad literaria. 